\title{
Investigation of the Local Mechanical Properties of the SAC Solder Joint with AFM
}

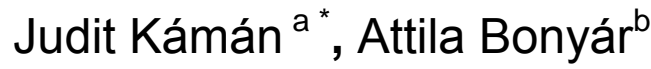 \\ Department of Electronics Technology \\ Budapest University of Technology and Economics \\ Budapest, Hungary

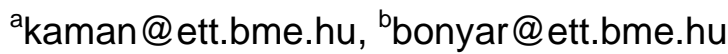

Keywords: Young's modulus, power dissipation, solder joint, AFM

\begin{abstract}
Considering the size of the natural appearance of the micro alloy components of a SAC solder joint, AFM was used to investigate their mechanical properties in the form of their natural appearance. Contact-mode point-spectroscopy was done to determine the elastic modulus and tapping-mode point-spectroscopy was done to investigate the tip-sample power dissipation.

The measured Young's modulus values of the $\mathrm{Cu}, \mathrm{IML}, \mathrm{Ag}_{3} \mathrm{Sn}$ and $\mathrm{Sn}$ components, were $125 \pm 9$ $\mathrm{GPa}, 111 \pm 20 \mathrm{GPa}, 67 \pm 11 \mathrm{GPa}$ and $57 \pm 16 \mathrm{GPa}$, respectively. The dissipation measurements were accomplished by $\mathrm{Si}$ and diamond probes with different spring constants. The different characteristics of the results are discussed.
\end{abstract}

\section{Introduction}

The Sn-Ag-Cu (SAC) alloys are the most commonly used lead-free substitute solders. The reliability of the solder-joints is heavily influenced by the characteristics of the formed intermetallic components (IMCs) during the soldering [1]. The following parts (phases) can be distinguished: the $\mathrm{Cu}$ substrate, the intermetallic layer (IML) which formed at the interface of the solder paste and the copper, the $\mathrm{Ag}_{3} \mathrm{Sn}$ islands, which grows up in the solder and the $\mathrm{Cu}_{6} \mathrm{Sn}_{5}$ islands which spall from the IML and migrate into the $\mathrm{Sn}$-rich phase $[2,3]$. The general nanoindentation techniques require thermal aging treatments or preparing specific bulk materials [4] to obtain sufficient thickness of the components. Nevertheless, the natural appearance of the microconstituents may differ from these artificially prepared forms.

However, Atomic Force Microscopy (AFM) was primarily developed to investigate the surface structures at nanometer scale, several other applications begun to spread to characterize the inhomogeneity in surface material. In this article two methods were applied, which are technically feasible with a basic AFM to investigate the mechanical properties of the sample. One is the Young's modulus determination by contact-mode point-spectroscopy; the other is the calculation of the tip-sample energy-dissipation using tapping-mode point-spectroscopy data.

\section{Theory}

Determination of Young's Modulus. To determine the Young's modulus, the Hertz-Sneddon model was used [5]. This model describes the relation between the loading force $(F)$ and the elastic sample deformation $(\delta)$ through Young's modulus $(E)$ assuming a conical indenter:

$$
F=\frac{2 E \tan (\alpha)}{\pi\left(1-v^{2}\right)} \delta^{2}
$$

where $\alpha$ is the half opening angle of the tip and $v$ is the Poisson-ratio.

During the contact-mode point-spectroscopy the absolute piezo position $(z)$ and the absolute cantilever deflection $(d)$ can be measured. Using Hook's law the loading force can be calculated by the deflection $(d)$ times the spring constant $(k)$ of the cantilever. The sample deformation equals to 
the difference of the piezo position and the indentation. Considering the above mentioned facts, the next formula can be fitted to the measured $z(d)$ curves:

$z=z_{0}+d+\sqrt{\frac{k\left(1-v^{2}\right) \pi}{2 E \tan (\alpha)}} \sqrt{d}=z_{0}+d+B \sqrt{d}$

where $z_{0}$ is the piezo position at the contact point and $B$ is the fitting parameter. As it can be seen in Eq. (1) the fitting parameter $B$ depends on the $E$ elastic modulus through the Poisson-ratio and the constants describing the tip. However the exact determination of these constants can be difficult.

The deviation of the parameter $B$ is strongly depends on the correct assignment of the elastic region. However several papers were published which presented an algorithm to find the tip-sample contact point $(\mathrm{CP})$-which could be regarded as the initial point of the fitting -, less attention was paid to determine the last point of the fitting and there is no widely accepted solution for this problem [6, 7]. In our work, considering the attractive part of the measured curves, the CP point was chosen as the minimum point of the curve and the last point of the fitting was determined by visual assessment.

Choosing tip with a convenient spring constant regarding the sample hardness is essential to get a force curve with a relevant elastic region.

Tip-Surface Power Dissipation. Using the model of Cleveland et al. [8] on the tapping mode point-spectroscopy data the average energy-dissipation of the tip per a cycle can be calculated. If we assume fix settings parameters during the measurements, this energy-dissipation can characterize the surface material of the sample.

Analyzing the energy balance of the vibrating tip and the sample, in the most simplified case the following equation describes the average power dissipation during the point-spectroscopy:

$\overline{P_{\text {tıp }}}=\frac{1}{2} \frac{k A^{2} \omega_{0}}{Q_{\text {cant }}}\left[\left(\frac{A_{0}}{A}\right) \sin (\varphi)-1\right]$

Besides the measured amplitude $(A)$ and phase-lag $(\varphi)$ compared to the drive only the properties of the cantilever are present in the equation, such as the spring constant $(k)$, the resonance frequency $\left(\omega_{0}\right)$ and the quality factor $\left(Q_{\text {cant }}\right) . A_{0}$ is the free amplitude when the tip is far from the surface and does not interact with the force-field of the sample.

\section{Methods and Materials}

SENJU SAC305 paste was soldered to a copper wire by a solder iron. To study the cross section of the solder joint the sample was embedded into an acryl based resin (Technovit 4006). First, wet grinding process was applied on it with the following grit sizes: 80, 320, 500 and 1200; after it was polished by 9,3 and $1 \mu \mathrm{m}$. To reveal the relevant microstructures Struers OP-S (Oxide Polishing Suspension) was used for the final polishing which etched selectively the components.

Veeco (lately Bruker) diInnova and Bruker Dimension Icon type atomic force microscopes were used for the measurements in contact- and tapping-mode with 512 x 512 sampling rate and $1 \mathrm{~Hz}$ scan rate. At each measurement the PID (Proportional-Integral-Derivative controller) values were optimized according to the user manual. Budget Sensors Tap150Al-G probes were used for the scanning and for the contact-mode point-spectroscopy. The tapping-mode point-spectroscopy measurements were done by Bruker Tap 150 and ART D160 probes. By the datasheet of the suppliers the nominal spring constant of each probe is $5 \mathrm{~N} / \mathrm{m}$, however it can vary within a large range, namely between $1.5 \mathrm{~N} / \mathrm{m}$ and $15 \mathrm{~N} / \mathrm{m}$. The nominal resonant frequency is $150 \mathrm{kHz}$ at each probe, but the precise values were obtained from the resonance curve during the tuning, such as the quality factor. The half opening angle of the tip $(\alpha)$ was considered as $20^{\circ}$ and $v=0.33$ was used as the Poisson-ratio. For the visualization Gwiddion 2.27 software was used.

EDS (energy dispersive X-ray analysis) were done with Bruker Quantax EDX system. 


\section{Results and discussions}

Determination of Young's Modulus. Due to the sample preparation the five different phases in the solder joint can be clearly distinguished in the topography maps; the copper, the intermetallic layer (IML), the tin-phase, the $\mathrm{Ag}_{3} \mathrm{Sn}$ formed in the tin and $\mathrm{Cu}_{6} \mathrm{Sn}_{5}$ spelled into the tin phase (see Fig 1.). EDS measurements showed that the IML consists of about $90 \% \mathrm{Cu}_{6} \mathrm{Sn}_{5}$ thus considering our goals only this phase was investigated in the IML. Also, it can clearly be seen in Fig. 1 that the size of the Ag3Sn phases is extremely small (diameter under $1 \mu \mathrm{m}$ ), which means that the investigation of this phase clearly would not be possible with classical micro-indentation techniques which cover much larger areas.
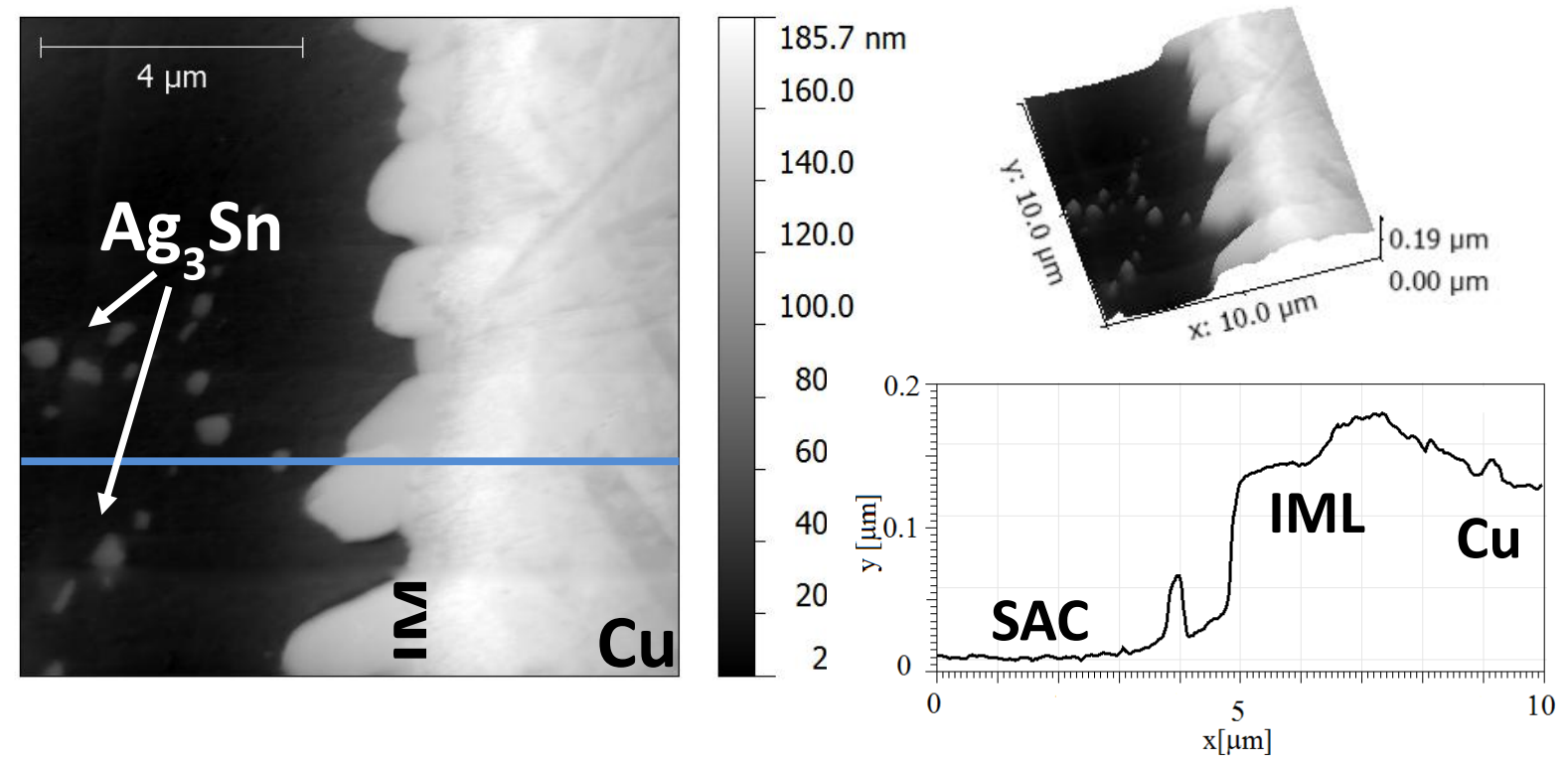

Fig. 1. $20 \mu \mathrm{m} \times 20 \mu \mathrm{m}$ tapping-mode topography image made on a SAC solder joint cross-section (left). The 3D representation (top right) and the cross-section profile at the indicated marker (bottom right) show, that the components are selectively etched by OP-S suspension.

To determine the Young's modulus, 7-7 point-spectroscopy measurements were done at each region to avoid the degradation of the tip. Fig. 2 presents the calculated Young's modulus values using Eq. (2) as a fitting function.

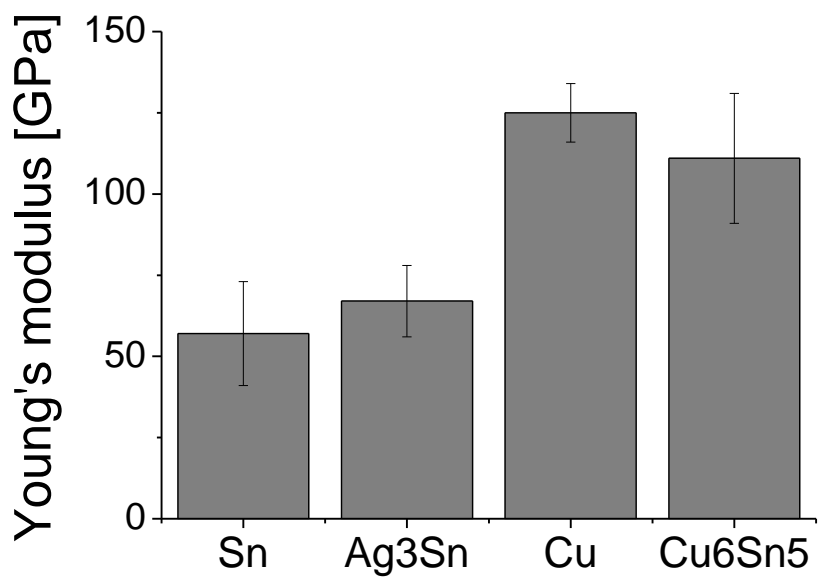

Fig. 2. The calculated Young's modulus values of the contact-mode point-spectroscopy measurements using Eq. (2) as a fitting function

It can be seen, that the elastic property of the $\mathrm{Ag}_{3} \mathrm{Sn}$ is similar to the $\mathrm{Sn}$ and the elastic behavior of the IML $\left(\mathrm{Cu}_{6} \mathrm{Sn}_{5}\right)$ is closer to the $\mathrm{Cu}$. The measured Young's modulus of the $\mathrm{Ag}_{3} \mathrm{Sn}$ and Sn were $67 \pm 11 \mathrm{GPa}$ and $57 \pm 16 \mathrm{GPa}$, respectively, while the other two components are significant harder, 
namely the $\mathrm{Cu}_{6} \mathrm{Sn}_{5}$ is $111 \pm 20 \mathrm{GPa}$ and the $\mathrm{Cu}$ is $125 \pm 9 \mathrm{GPa}$. The published values of the Young's modulus of the intermetallic components vary in a similar range as the deviation of our measurements [4, 9- 11]. Although our method is basically different from the classical indentation techniques, these values correlate well with the published results. The advantage of this method is that it does not require thermal aging or bulk samples, but the results can have larger deviation. Several factors can contribute to the deviation, such as the surface inequalities. If the tip contacts the surface in angles other than $90^{\circ}$, the torture of the cantilever can modify the deflection signal and the effective contact area of the tip can also change.

Tip-Surface Power Dissipation. Silicon and diamond probes were used to characterize the microstructural components with the tip-surface dissipation method. 10-10 tapping-mode pointspectroscopy curves were done at each region with each probe. Typical amplitude and phase curves of the tapping-mode point spectroscopy can be seen in the Fig. 3 with the calculated dissipation curve. During the measurements the setting parameters were tried to be maintained constant, since the dissipation values necessarily depend on the amount of the initial power fed into the cantilever. For the calculation, $148.35 \mathrm{kHz}$ and $131.75 \mathrm{kHz}$ were used as the resonant frequency and $Q_{\text {cant }}=178$ and $Q_{\text {cant }}=82$ were obtained as the quality factor based on the resonance curves for the silicon and the diamond tips, respectively.

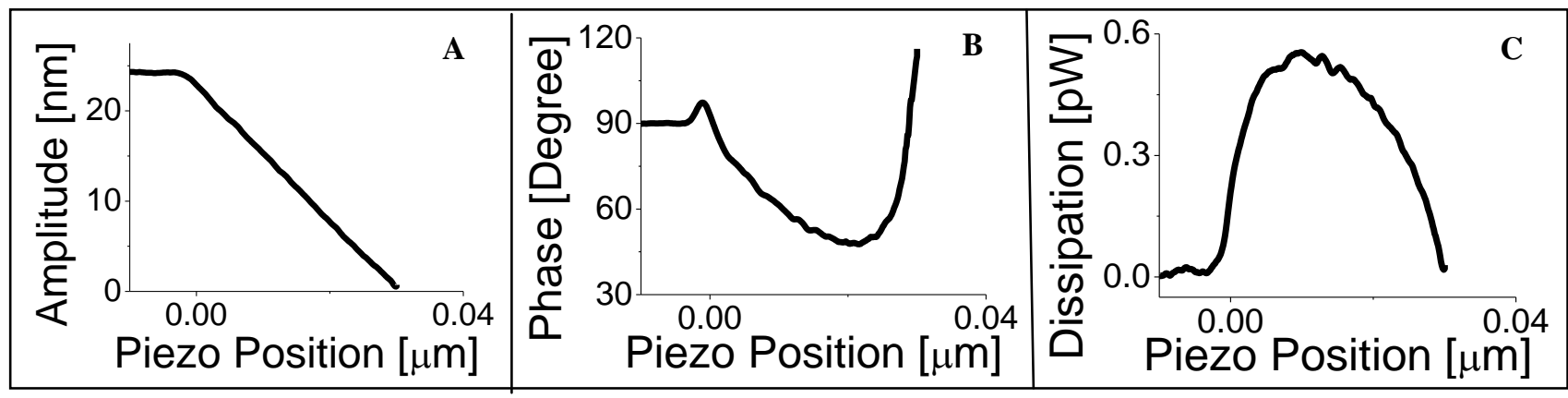

Fig. 3. Measured amplitude (A) and phase (B) curves obtained by tapping-mode point-spectroscopy and the calculated dissipation curve $(\mathrm{C})$ at $\mathrm{Cu}$ substrate

The maximum of the dissipation curve is regarded as the dissipation value belonging to the material. Table 1 and Fig. 4 show the mean of the calculated dissipations for both probes. It shows different tendencies for the different types of probes. In case of the Si probe, the dissipation correlates with the Young's modulus and it shows increasing tendencies. In case of the diamond probe, the dissipation has a minimum at $\mathrm{Cu}$.

Table 1. The calculated dissipation values of the different phases in SAC305

\begin{tabular}{|c|c|c|}
\hline Component & $\begin{array}{c}\text { Dissipation [pW] } \\
\text { using Si probe }\end{array}$ & $\begin{array}{c}\text { Dissipation [pW] } \\
\text { using diamond probe }\end{array}$ \\
\hline $\mathrm{Sn}$ & $0.34 \pm 0.032$ & $0.68 \pm 0.037$ \\
$\mathrm{Ag}_{3} \mathrm{Sn}$ & $0.50 \pm 0.034$ & $0.65 \pm 0.026$ \\
$\mathrm{Cu}$ & $0.52 \pm 0.012$ & $0.55 \pm 0.028$ \\
$\mathrm{Cu}_{6} \mathrm{Sn}_{5}$ & $0.62 \pm 0.023$ & $0.62 \pm 0.022$ \\
\hline
\end{tabular}




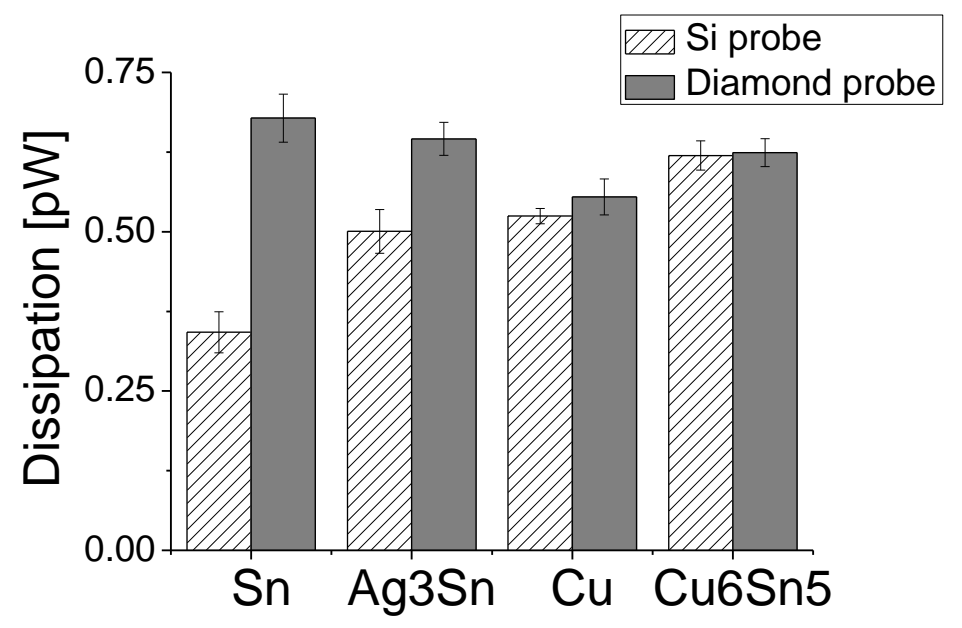

Fig. 4. Comparison of maximum dissipation values of the solder joint components measured by silicon and diamond probe

Based on the results we can see that the obtained dissipation values and the relation between these values and the elastic/hardness properties of the investigated material is strongly depending on the used cantilever. Based on our other current investigations on a wider range of samples (data not shown) we suspect that the relation between the elastic properties of the cantilever and the elastic/hardness properties of the investigated material determines the obtained dissipation values. We also suspect that for a given cantilever with known $k$, the obtained dissipation vs. elastic modulus curve has a definite minimum, which is depending on the $k$ of the cantilever. In Fig. 4 this postulated minimum would be around the $\mathrm{Cu}$ for the diamond probe and is shifted to the left around - or even below - Sn for the Si probe. Based on thermal tuning by Icon AFM we assume that the Si probe has a significantly smaller $k$. Data regarding these investigations will be presented elsewhere.

\section{Summary}

Contact-mode point-spectroscopy and tip-surface energy dissipation measurements were done with AFM to measure the surface mechanical properties of micro alloy components in a SAC solder joint sample. The obtained Young's modulus values correlates well with the previously reported values obtained by using classical indentation techniques. For the best of our knowledge, this was the first time that the elasticity of the fine $\mathrm{Ag}_{3} \mathrm{Sn}$ component in the SAC solder was measured in its natural appearance. The tip-surface energy dissipation measurements show, that the obtained dissipation values are greatly depending on the elastic properties of the utilized cantilever. Here more investigations are needed to determine the connection between the elastic/hardness properties of the sample and the measured tip-surface dissipation.

\section{Acknowledgement}

Judit Kámán thanks the support of Nanofabrication and Nanocharacterization Core Facility of CEITEC open access project, ID number LM2011020, funded by the Ministry of Education, Youth and Sports of the Czech Republic under the activity „Projects of major infrastructures for research, development and innovations".

\section{References}

[1] S.-K. Seo, M. G. Cho, H. M. Lee, W. K. Choi, Comparison of Sn2.8Ag20In and Sn10Bi10In Solders for Intermediate-Step Soldering, J. Electron. Mater. 3511 (2006) 1975-1981. 
[2] M. G. Cho, S. K. Kang, D-Y. Shih, H. M. Lee, Effects of Minor Additions of Zn in Interfacial Reactions of Sn-Ag-Cu and $\mathrm{Sn}-\mathrm{Cu}$ Solders with Various $\mathrm{Cu}$ Substrates during Thermal Aging, J. Electron. Mater. 35, 11 ( 2007) 1501-1509.

[3] Y. C. Sohn, J. Yu, S. K. Kang, D. Y. Shih, T. Y. Lee, Spalling of Intermetallic Compounds During the Reaction Between Lead-Free Solders and Electroless Ni-P Metallization, J. Mat. Res.19 8 (2011) 2428-2436.

[4] X. Deng, M. Koopman, N. Chawla, K. K. Chawla, Young's Modulus of (Cu, Ag)-Sn Intermetallics Measured by Nanoindentation, Mater. Sci. Eng. 364 1-2 (2004) 240-243.

[5] J.Domke, M. Radmacher. Measuring the Elastic Properties of Thin Polymer Films with the AFM, Langmuir 14 (1998) 3320-3325.

[6] K. A. Melzak, S. Moreno-Flores, K. Yu, J. Kizhakkedathu, J. L. Toca-Herrera, Rationalized Approach to the Determination of Contact Point in Force-Distance Curves: Application to Polymer Brushes in Salt Solutions and in Water, Micros. Res. Tech. 7310 ( 2010) 959-964.

[7] S. L. Crick, F. C. P. Yin, Assessing Micromechanical Properties of Cells with Atomic Force Microscopy: Importance of the Contact Point, Biomech. Model. Mechanobiol. 63 (2007) 199-210.

[8] J. P. Cleveland, B. Anczykowski, A. E. Schmid, and V. B. Elings, Energy Dissipation in Tapping-Mode Atomic Force Microscopy, Appl. Phys. Lett. 7220 (1998) 2613-2615.

[9] S. Lotfian, J. M. Molina-Aldareguia, K. E. Yazzie, J. Llorca, N. Chawla, Mechanical Characterization of Lead-Free Sn-Ag-Cu Solder Joints by High-Temperature Nanoindentation, J. Electron. Mater. 426 (2013) 1085-1091.

[10] Y. Sun, J. Liang, Z.-H. Xu, G. Wang, X. Li, Nanoindentation for Measuring Individual Phase Mechanical Properties of Lead Free Solder Alloy, J. Mater. Sci. -Mater. El. 196 (2008) 514-521.

[11] R. R. Chromic, R. P. Vinci, S. L. Allen, M. R. Notis, Measuring the Mechanical Properties of $\mathrm{Pb}-$ Free Solder and Sn-Based Intermetallics by Nanoindentation, JOM -J. Min. Met. Mat. S. 556 (2003) 66-69. 